\title{
A Review of Mindfulness-Based Interventions in the Treatment of Substance Abuse and Addiction
}

\author{
Huang Man ${ }^{1}$, Zhang Jun ${ }^{2}$, Zhang Siying ${ }^{3 *}$ \\ ${ }^{123}$ Hunan Judicial Police Vocational College, Changsha, Hunan 410131 \\ 1352819709@qq.com
}

\begin{abstract}
As a treatment for substance abuse and addiction, mindfulness-based interventions(MBI) is the third major treatment method emerging after behavioral therapy and cognitive behavioral therapy. Its key is changing cognitive, emotional and behavioral processes. MBI focuses on "cultivating the conscious experience that includes the same cognition, as well as non-judgmental conscious, emotional and behavioral self-regulating processes. " This article aims to show the effects of mindfulness-based interventions on substance abuse and substance addiction by reviewing previous related research. It summarizes the impact mechanism of mindfulness-based interventions to provide information for clinical practice and directions for future research. Keywords: mindfulness-based interventions, substance abuse and addiction, influence mechanism, clinical practice
\end{abstract}

\section{正念干预对物质滥用与成㒣治疗的研究综述}

\author{
黄曼 ${ }^{1}$, 张军 ${ }^{2}$, 张思盈 ${ }^{3 *}$ \\ 123 湖南司法警官职业学院 湖南长沙 410131 \\ 1352819709@qq.com
}

\section{摘要}

正念干预 (MBI) 作为治疗物质滥用与成瘾是在行为疗法和认知行为疗法而后兴起的第三大治疗方法, 其重点在于改变认知, 情绪和行为过程, MBI 专注于 “培养对包含同样认知的意识体验的经验和意识的 非判断意识, 情绪和行为自我调节过程”。本文的目的是回顾关于正念干预治疗物质滥用和物质成瘾疗 效的证据及以往相关的研究分析, 总结正念干预的影响机制, 旨在为临床实践提供资讯, 并为未来研 究提供方向。

关键词：正念干预 物质滥用与成瘾 影响机制 临床实践

\section{1. 正念干预与物质滥用与成瘾}

尽管物质滥用和成瘾的治疗方法很多, 但与传统治 疗相关的临床结果仍然不能令人满意。例如, 认知行为 疗法已证明对几种类型的物质滥用和物质成瘾具有显 着的效果（Irvin, Bowers, Dunn, \& Wang, 1999）。然而, 尽管这种方法取得了短期成功, 但复发仍然是认知行为 疗法干预的 $50-70 \%$ 物质滥用和物质成瘾患者的一个重 要问题（McHugh, Hearon, \& Otto, 2010）。如果考虑到 物质滥用和物质成瘾治疗后的复发率仍然高达 $60 \%$

(Connors, Maisto, \& Donovan, 1996; United Nations Office of Drugs and Crime, 2007), 需要有效治疗在加强 减少物质使用和预防未来复发方面变得明显。因此, 在 过去几十年中, 寻求可以成功减少物质使用和复发率的
新干预措施已经获得了越来越多的关注。特别是，基于 正念的干预措施越来越多地被提倡作为潜在的干预方 法（Marlatt \& Chawla, 2007; Zgierska et al., 2009）。

正念是一种系统的发展, 关注当下的经验, 接受和 不评判的态度 ( Bishop et al, 2004; KabatZinn, 1994)。 此外, 正念冥想最初源于佛教内观禅修, 是 KabatZinn 于 1979 年开发的“正念减压疗法”（MBSR）的基石，旨 在教导患有慢性身心健康问题的患者如何改善他们的 生活, 但在过去的几十年里, 它也被证明是临床心理学 中一种治疗慢性身心疾病卓有成效的手段 (Chiesa \& Serretti, 2010; Hofmann \& Sawyer, 2010）。

最近, 越来越多的研究指出正念干预可以帮助人们 发现并解决物质滥用与成瘾相关的问题 (Zgierska et al, 2009）。正念干预包括广泛的干预措施, 目前尚不完全 清楚它们彼此的差异程度 (Chiesa \& Malinowski, 2011)。 


\subsection{3 非评判地关注}

对一个人的经历进行评判被视为具有放大其影响 的倾向, 而不是评估我们的认知和情感体验, 正念教会 我们简单地注意它们。通过减少习惯倾向来对经验进行 二分类分类, 正念被视为增强了认知和行为行为的可用 范围和适应性（Hayes \&Wilson, 2003）。这种增强的“认 知灵活性” (Roemer \& Orsillo, 2003) 增加了对经验的开 放性, 并减少了将某些经历标记为“敌人”的北向。

\subsection{4 认知疗法和正念干预之间的差异}

虽然一些基于正念的干预措施的运作方式与传统 的认知一行为疗法相似, 但通过关注思维的偏离 ( “思 想不等于现实”），当我们考虑对特定认知的反应时, 就会出现差异。正念干预旨在改变认知过程, 而不是内 容（Orsillo et al, 2004）。

\section{2. 生理机制}

从生理的角度来看待正念干预的影响机制, 重要的 是要看心理变化是否与生理和生化变化相关。Davidson (2003) 进行了一项随机对照研究, 初学冥想者完成了 为期 8 周的正念减压课程, 与对照组相比, 正念减压组 在接触后 4 周和 8 周的评估中证实前皮质的左侧激活增 加和抗体滴度增加。在某些形式的积极情绪表达期间观 察到前皮层区域的左侧激活, 并且在抑有的人中通常是 不活跃的 (Davidson et al, 2000)。这些研究结果表明正 念认知疗法可能影响弱势个体的抑郁相关生理。同时有 研究者比较了经验丰富的冥想练习者和非冥想者, 发现 在进行同情冥想时, 经验丰富的冥想练习者产生了更大 的持续脑电图 (EEG) 伽马带振荡和相位同步 (Lutz, 2004）。脑电图中的这种变化表明, 在这种冥想状态期 间，有经验的冥想者的神经活动异常协调。

\section{3. 总结与启发}

物质滥用与成滤, 包括吸毒成瘾是生理因素、心理 因素和社会因素三者共同作用的结果, 尤其是受错误认 知影响。我们将在戒毒人员的防复吸研究中主要将“正 念”的思维与理念有效植入戒毒人员的脑海和生活中, 进一步挖掘吸毒人员在戒毒中的错误认知思维, 促使戒 毒人员的心理模式能够及时换挡, 从而有效促进吸毒人 员认知改变, 使吸毒成员能够自我认知吸毒带来的严重 危害、自觉改变毒品错误认知、自我弄清愉快需求错位 的成㒣本质、自觉内化纠正错位需求回归正常需求的戒 毒信念、自觉强化摆脱毒瘾纠细恢复健康人生的戒毒动 机、自主抵御纠正错位嗜毒欣快需求, 自主持续坚持用 正常愉快替代吸毒欣快。最终使戒毒人员能够自觉的厌 恶吸毒、放弃吸毒、反对吸毒。 


\section{REFERENCES}

[1] Black, D. S. (2014). Mindfulness-based interventions: an antidote to suffering in the context of substance use, misuse, and addiction. Substance use \& misuse, 49(5), 487-491.

[2] Brewer, J. A., Elwafi, H. M., \& Davis, J. H. (2014). Craving to quit: Psychological models and neurobiological mechanisms of mindfulness training as treatment for addictions.

[3] Bishop, S. R., Lau, M., Shapiro, S., Carlson, L., Anderson, N. D., Carmody, J., ... \& Devins, G. (2004). Mindfulness: A proposed operational definition. Clinical psychology: Science and practice, 11(3), 230-241.

[4] Brown, K. W., \& Ryan, R. M. (2003). The benefits of being present: mindfulness and its role in psychological well-being. Journal of personality and social psychology, 84(4), 822. 